\title{
Treatment of Epidermal Growth Factor (EGF) enhances Nuclear Maturation of Porcine Oocytes and Stimulates Expression of ER/Golgi Transport Proteins
}

\author{
Yong Hwangbo ${ }^{1^{*}}$, Hae-In Oh${ }^{1 *}$, Sang-Hee Lee ${ }^{2}$, Hee-Tae Cheong ${ }^{3}$, \\ Boo-Keun Yang ${ }^{1}$ and ${ }^{\dagger}$ Choon-Keun Park ${ }^{1}$ \\ ${ }^{1}$ College of Animal Life Sciences, Kangwon National University, Chuncheon 24341, Republic of Korea \\ ${ }^{2}$ Institute of Animal Resources, Kangwon National University, Chuncheon 24341, Republic of Korea \\ ${ }^{3}$ College of Veterinary Medicine, Kangwon National University, Chuncheon 24341, Republic of Korea
}

\begin{abstract}
This study was conducted to investigate stimulatory effect of epidermal growth factor (EGF) on nuclear maturation and the expression level of EGF-receptor (EGFR), GM-130 (a marker of Golgi apparatus), transport protein Sec61 subunit beta (Sec61ß), and coatomer protein complex subunit gamma 2 (COPG2) in porcine oocytes. The cumulus-oocyte complexes were collected from follicle with 3-6 mm in diameter. They were incubated in medium with/without EGF for $22 \mathrm{~h}$ (IVM I ) and subsequently incubated hormone-free medium with/without EGF for $22 \mathrm{~h}$ (IVM II ). Nuclear maturation state was checked by aceto-orcein stain. Protein expression of EGFR, GM-130, Sec61 $\beta$, and COPG2 were measured by immunofluorescence. In results, nuclear maturation of oocytes in EGF non-treated oocytes were significantly lower than EGF-treated groups at IVM I or IVM II stage $(P<0.05)$, whereas maturational rate in EGF treatment groups at both of IVM stage was higher in among the all treatment groups $(P<0.05)$. EGFR, GM-130, Sec61 $\beta$ and COPG2 were expressed in the cytoplasm of oocytes. Especially, GM-130 and EGFR were strongly expressed, but Sec61 $\beta$ and COPG2 were weakly expressed in cortical area of cytoplasm. The protein level of GM-130, Sec61 $\beta$, and COPG2 were significantly higher in the EGF-treated groups $(P<0.05)$. However EGFR was no difference between non EGF-treated groups and control. In conclusion, EGF plays an important role in the systems for oocyte maturation with endoplasmic reticulum and Golgi apparatus. In addition, the protein levels of Sec61 $\beta$ and COPG2 could be changed by EGF in the porcine oocytes during maturation.
\end{abstract}

Key words : Porcine oocyte, In vitro maturation, Epidermal growth factor, Transport protein

\section{INTRODUCTION}

Generally, first resumption and completion of meiosis in mammalian oocytes are essential process for capacity of fertilization and developmental competence after oocytes maturation (Eppig, 1996). In addition, nuclear and cyto- plasmic maturation of oocytes are involved with activation of oocyte, fertilization process, and embryo development before implantation (Eppig et al., 1994), and it require sufficient nutrients from follicular fluid and cells (Steeves \& Gardner, 1999; Sutton-McDowall et al., 2005). Moreover, a variety of growth factors and hormones that are secreted

\footnotetext{
Manuscript received April 6, 2017, Received in revised form April 10, 2017, Accepted April 14, 2017

$\dagger$ Corresponding Author : Choon-Keun Park, College of Animal Life Science, Kangwon National University, Chuncheon 24341, Republic of Korea. Tel. : +82-33-250-8627, Fax : +82-33-250-5574, E-mail : parkck@kangwon.ac.kr

* Both authors contributed equally in this work.

This is an Open Access article distributed under the terms of the Creative Commons Attribution Non-Commercial License (http:// creativecommons.org/licenses/by-nc/3.0) which permits unrestricted non-commercial use, distribution, and reproduction in any medium, provided the original work is properly cited.
} 
from follicular cells stimulated both of nuclear and cytoplasmic maturation of oocytes (Mattioli et al., 1988; Procházka et al., 2000).

For successful oocyte maturation and subsequent development, cellular organelles in cytoplasm of oocytes undergo a physiological changes during maturation. Especially, the Golgi apparatus and endoplasmic reticulum (ER) are closely related with cytoplasmic and nuclear maturation. The Golgi apparatus, also known as Golgi complex, is involved with various intracellular trafficking events and ER plays role in protein folding, degradation, and store of calcium ion (Mao et al., 2014). These organelles of oocytes are redistributed in cytoplasm of oocytes and undergone the structural changes during maturation. And interaction between Golgi apparatus and ER is an important for both of cytoplasmic and nuclear maturation. Moreno et al. (2002) had reported that treatment of brefeldin A, inhibitor of membrane trafficking, during in vitro maturation of mouse oocyte blocked nuclear maturation and this study indicated that membrane trafficking from ER to the Golgi apparatus is closely associated with oocyte maturation. As a one of transport protein, transport protein Sec61 subunit beta $(\operatorname{Sec} 61 \beta)$ is an important factor of the protein translocation of ER membrane and coatomer protein complex subunit gamma 2 (COPG2), a one of type-1 COP, is involved with retrograde transport of protein from Golgi apparatus to ER (Mendis et al., 2008). These reports suggested that Sec61 $\beta$ and COPG2 are concerned with oocyte maturation through regulation of protein transport between Golgi apparatus and ER, however, roles of transport protein on oocyte maturation were not fully understood.

As a one of growth factor, epidermal growth factor (EGF) plays an important role in cell growth, proliferation, and differentiation by stimulation of mitosis in various type of cells (Yarden, 2001). And it also induces resumption of meiosis in mammalian oocytes (Bolamba et al., 2006; Lindbloom et al., 2008). This growth factor was contained in mammalian follicular fluids including pigs
(Conti et al., 2006). Uhm et al. (2010) had reported that nuclear maturation of porcine oocytes were stimulated by action of EGF instead of follicle-stimulating hormone (FSH). Stimulatory effects of EGF on cellular process were generated by binding into its specific receptor (EGFR) and interaction between EGF and EGFR induced intra-cellular signal transduction, including mitogen-activated protein kinase (MAPK) and extracellular signal-regulated kinase (ERK)(Filardo et al., 2002). Because a variety of signal pathways are related with oocyte maturation, biolo-gical function of EGF is an important for successful matu-ration of mammalian oocytes. Therefore, we expected that EGF stimulates porcine oocyte maturation via regulation of transport protein, and the aim of this study was to investigate stimulatory effect of EGF on nuclear maturation and expression of Sec61 $\beta$ and COPG 2 in porcine oocytes.

\section{MATERIALS AND METHODS}

\section{In vitro maturation (IVM)}

All procedures that involved the use of animals were approved by the Kangwon National University Institutional Animal Care and Use Committee (KIACUC-090139). Porcine ovaries were collected from slaughtered gilts at local slaughter house and transferred to the laboratory in $0.9 \%(\mathrm{w} / \mathrm{v})$ sterilized saline within $2 \mathrm{~h}$. The cumulusoocyte complexes (COCs) were aspirated from antral follicles with 3-6 $\mathrm{mm}$ in diameter using $10 \mathrm{cc}$ syringe with 18-gauge needle. After aspiration, COCs with homogeneous cytoplasm and compact cumulus layer were selected. To investigate effect of EGF on nuclear maturation of porcine oocyte, COCs were maturated in medium-199 (Invitrogen, MA, USA) containing $10 \%(\mathrm{v} / \mathrm{v})$ porcine follicular fluid (pFF), $10 \mathrm{IU} / \mathrm{mL}$ human chorionic gonadotropin (hCG; Intervet), $10 \mathrm{ng} / \mathrm{mL}$ luteinizing hormone (LH; Sigma-Aldrich, St. Louis, MO, USA), $10 \mathrm{ng} / \mathrm{mL}$ follicle stimulating hormone (FSH; Sigma-Aldrich) with or without $10 \mathrm{ng} / \mathrm{mL}$ EGF (Sigma-Aldrich) at $38.5^{\circ} \mathrm{C}, 5 \% \mathrm{CO}_{2}$ 
condition for $22 \mathrm{~h}$ (IVM- I ) and subsequently maturated using hormone-free medium-199 containing 10\% (v/v) $\mathrm{pFF}$ with or without $10 \mathrm{ng} / \mathrm{mL}$ EGF for $22 \mathrm{~h}$ (IVM- II ).

\section{Evaluation of nuclear maturation}

Aceto-orcein stain method was used to evaluate nuclear maturation stage of porcine oocyte. Incubated COCs were treated by $0.1 \%(\mathrm{v} / \mathrm{v})$ hyaluronidase. Cumulus cells surrounding zona pellucida of oocyte were removed by gentle pipetting using pasteur pipette. Denuded oocytes were fixed in acetic alcohol solution (acetic acid : ethanol; $1: 3$; $\mathrm{v} / \mathrm{v}$ ) for $48 \mathrm{~h}$, at room temperature (RT), and nuclear was stained by $1 \%(\mathrm{w} / \mathrm{v})$ aceto-orecein at RT for $7 \mathrm{~min}$. The morphology of nuclear was observed under light microscope. The oocytes at germinal vesicle (GV), germinal vesicle breakdown (GVBD), metaphase I (M I ), and telophase I (T I ) phase were decided as immature oocyte and MII stage oocytes were classified as mature oocyte.

\section{Immunofluorescence}

Mature oocytes, which were incubated with or without EGF during IVM stage, were denuded and fixed in $4 \%$ (v/v) paraformaldehyde solution at $4{ }^{\circ} \mathrm{C}$ for $1 \mathrm{~h}$. For permeabilization of oocytes, PBS containing $0.5 \%(\mathrm{v} / \mathrm{v})$ Triton X-100 (Sigma-Aldrich) was used. Then, blocking process of oocytes was carried out using blocking solution [PBS containing $0.1 \mathrm{M}$ glycine, $0.01 \%$ (v/v) Triton X-100, $1 \%(\mathrm{w} / \mathrm{v})$ non-fat dry milk, $0.5 \%(\mathrm{w} / \mathrm{v})$ bovine serum albumin, and $0.02 \%(\mathrm{w} / \mathrm{v})$ sodium azide] at RT during overnight. Immunofluorescence was conducted using goat anti-COPG2 (sc-23204, 1:500, Santa Cruz, USA), EGFR (sc-31156, 1:1000, Santa Cruz), rabbit anti-Sec61ß (PA3015, 1:1000, Thermo Fisher scientific, Waltham, MA, USA), and GM130/GOLGA2 (maker of Golgi apparatus, NB110-57012, 1:100, Novus Biologicals, Littleton, CO, USA) at $4{ }^{\circ} \mathrm{C}$ during overnight and oocytes were incubated with goat anti-rabbit $\operatorname{IgG}(\mathrm{H}+\mathrm{L})$-Alexa Fluor 546 (A11010,
1:1,000, Invitrogen) and donkey anti-goat $\operatorname{IgG}(\mathrm{H}+\mathrm{L})$-Alexa Fluor 488 at $4{ }^{\circ} \mathrm{C}$ during overnight. The nuclear was counterstained by $1 \mathrm{ng} / \mathrm{mL}$ Hoechst 33342. For measurement of fluorescent intensity, immunofluorescence was conducted to 3 replication (40-50 oocytes per replication) and fluorescence images were captured using confocal laser scanning microscope (Olympus LX70 FV00 05-LPG-193, OLYMPUS, Japan) at same exposure time and photo multiplier tube (PMT). Fluorescence intensity was ana-lyzed by Multi gauge software ver 3.0 (Fuji photo Film, Japan).

\section{Statistical analysis}

All numerical data representing each parameter were analyzed using the Statistical Analysis System Software (SAS, version 9.3). Data are presented means \pm SEM and Duncan's multiple range tests. Comparisons among treatment groups were conducted using a generalized linear model (GLM) in the SAS package. A value of $P<0.05$ was considered to indicate a statistically significant difference.

\section{RESULTS}

The effect of EGF on nuclear maturation of porcine oocytes during IVM were presented in Table 1. Treatment of EGF during both of IVM stage enhanced the nuclear maturation rate of oocyte compare with other groups $(P<0.05)$. However, the portions of matured oocytes were lower in treatment groups that are absence of EGF during IVM I or IVM $\Pi$ stage. And it was lowest in EGF absence group among the all treatment groups $(P<0.05)$. In nonEGF stimulation group, immature oocytes were arrested mainly at GV and GVBD stage, especially, proportion of GV stage oocytes were higher than EGF-treated group during both IVM stage.

The expression of EGFR was detected on cytoplasm in both of EGF-treated and EGF non-treated oocyte (Fig. 1A). Fluorescence intensity in EGF treatment group was higher than non-treatment group, however, there was no signifi- 
cant difference (Fig. 1B). The Golgi apparatus was placed mainly in cytoplasm, whereas it was not located in sur- rounding chromosome region (Fig 2A). The expression level of the Golgi was stimulated by EGF treatment during

Table 1. Effect of epidermal growth factor on nuclear maturation of porcine oocytes during different in vitro maturation condition

\begin{tabular}{|c|c|c|c|}
\hline \multirow{2}{*}{$\begin{array}{c}\text { EGF treatment } \\
\left({ }^{*} \mathrm{IVM} \mathrm{I} /{ }^{* *} \mathrm{IVM} \amalg\right)\end{array}$} & \multirow{2}{*}{$\begin{array}{l}\text { No. of } \\
\text { oocytes }\end{array}$} & \multicolumn{2}{|c|}{ No. of (\%) } \\
\hline & & ${ }^{\text {aImmature oocyte }}$ & ${ }^{b}$ Mature oocyte \\
\hline$+/+$ & 130 & $52(40.0 \pm 2.2)^{\mathrm{c}}$ & $78(60.0 \pm 2.2)^{\mathrm{e}}$ \\
\hline$+/-$ & 148 & $100(67.6 \pm 0.4)^{\mathrm{d}}$ & $48(32.4 \pm 0.4)^{\mathrm{d}}$ \\
\hline$-1+$ & 137 & $100(73.0 \pm 7.4)^{\mathrm{d}}$ & $37(27.0 \pm 7.4)^{\mathrm{d}}$ \\
\hline$-1-$ & 136 & $118(86.8 \pm 7.3)^{\mathrm{e}}$ & $18(13.2 \pm 7.3)^{\mathrm{c}}$ \\
\hline
\end{tabular}

The data are present as mean \pm SEM from 3 replicates.

${ }^{a}$ Immature oocytes include germinal vesicle, germinal vesicle breakdown, metaphase, and telophase I phase oocytes.

${ }^{\mathrm{b}}$ Mature oocytes include only metaphase II oocytes.

${ }^{c-e}$ Different superscript in the same column indicate significant difference $(P<0.05)$.

* A period that is first 22 hour at in vitro maturation (IVM).

** Subsequent maturation stage for 22 hour after IVM I .

(A)

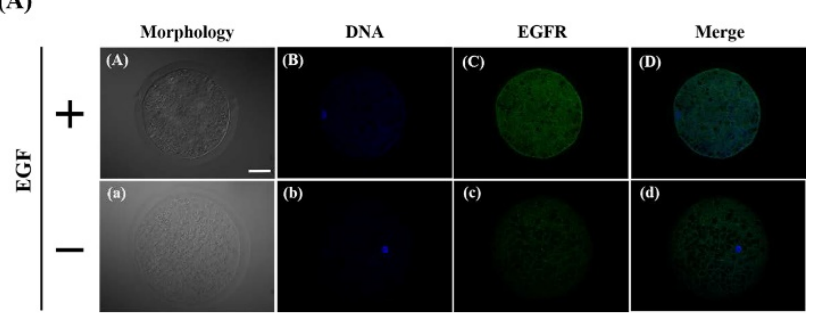

(B)

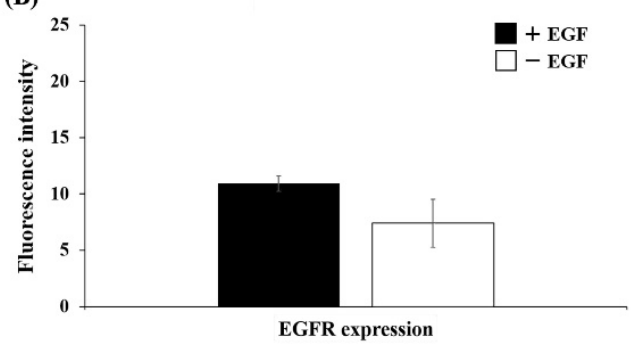

Fig. 1. Effect of epidermal growth factor (EGF) on expression of EGF receptor (EGFR) in porcine oocyte during in vitro maturation. (A) Immunofluorescence image of EGFR in maturate porcine oocyte with/without EGF (scale bar: $50 \mu \mathrm{m}$ ), (B) comparison of fluorescence intensity.
(A)

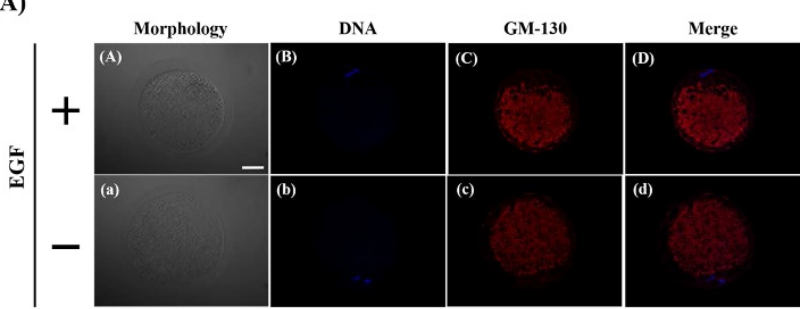

(B)

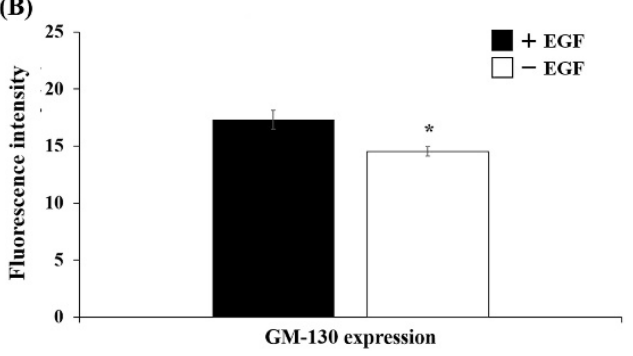

Fig. 2. Effect of epidermal growth factor (EGF) on expression of GM-130 in porcine oocyte during in vitro maturation. (A) Immunofluorescence image of GM-130 in maturate porcine oocyte with/ without EGF (scale bar: $50 \mu \mathrm{m}$ ), (B) comparison of fluorescence intensity. Asterisk indicate significant difference $(P<0.05)$. 
maturation compare with non-EGF group (Fig. 2B, $P<$ 0.05). Similar with EGFR expression, the COPG2 protein was detected on cortical area and cytoplasm (Fig. 3A). And fluorescent intensity of this protein was enhanced by EGF stimulation as well as Golgi apparatus expression (Fig. 3B, $P<0.05)$. Sec61 $\beta$ in both of treated and non-treated oocyte was distributed in cytoplasm and fluorescence intensity was increased in EGF-treated oocyte (Fig. 4, $P<0.05$ ).

\section{DISCUSSION}

This study was conducted to confirm the change of nuclear maturation of porcine oocytes by stimulation of EGF during IVM and we expected that it was mediated transport protein including COPG2 and Sec61 $\beta$. Nuclear maturational rate was enhanced by EGF treatment during both IVM stage and expression of Golgi apparatus, COPG2, and Sec61 $\beta$ were up-regulated in cytoplasm by EGF stimula-

(A)

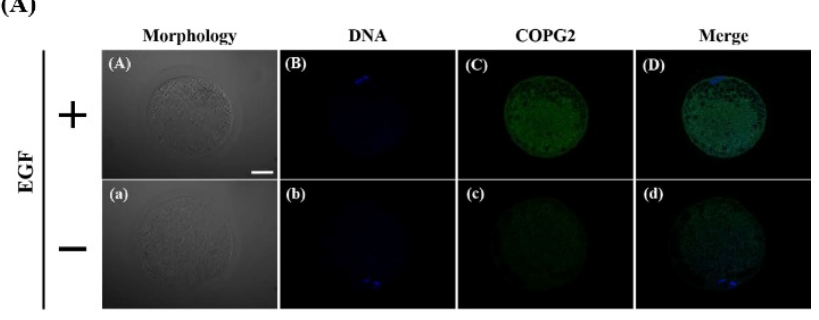

(B)

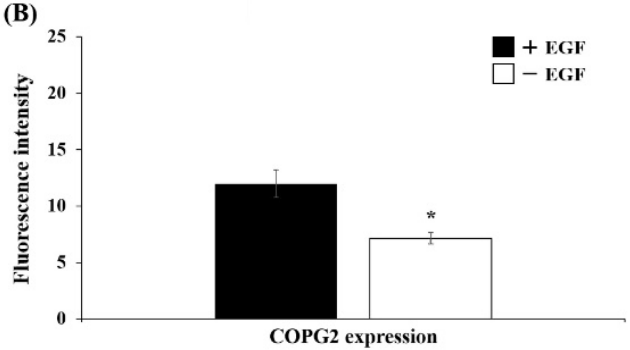

Fig. 3. Effect of epidermal growth factor (EGF) on expression of Sec61 subunit beta (Sec61ß) in porcine oocyte during in vitro maturation. (A) Immunofluorescence image of $\operatorname{Sec} 61 \beta$ in maturate porcine oocyte with/without EGF (scale bar: 50 $\mu \mathrm{m})$, (B) comparison of fluorescence intensity. Asterisk indicate significant difference $(P<0.05)$. tion.

Signal transduction by interaction between EGF and its specific receptor plays a crucial role in maturation process of mammalian oocytes. EGF and EGF-like ligands, such as amphoregulin, epiregulin, and transforming growth factor$\alpha$, shared and bind to the EGFR (Shimada et al., 2006). And these action improved the maturation of oocytes in various species including cows (Lonergan et al., 1996), mice (De La Fuente et al., 1999), monkeys (De Prada et al., 2009), and pigs (Coskun \& Lin, 1995). Coskun and Lin (1995) had reported that stimulation of porcine oocyte maturation by EGF is independent of the cyclic AMP (cAMP) and it might be related with protein kinase $\mathrm{C}$ (PKC) pathway. In this present study, nuclear maturation of porcine oocytes were enhanced by EGF stimulation at IVM stage, and expression of EGFR was increased in cytoplasm of EGF-treated oocytes. In the goat COCs, EGFR was localized

(A)

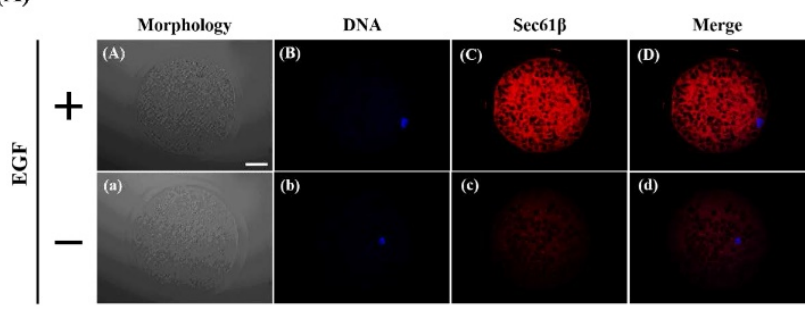

(B)

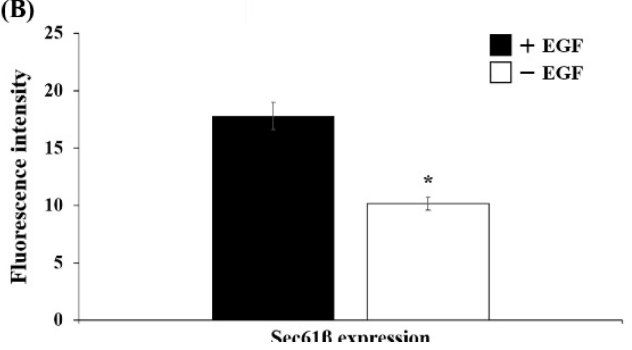

Fig. 4. Effect of epidermal growth factor (EGF) on expression of coatomer protein complex subunit gamma 2 (COPG2) in porcine oocyte during in vitro maturation. (A) Immunofluorescence image of COPG2 in maturate porcine oocyte with/without EGF (scale bar: $50 \mu \mathrm{m}$ ), (B) comparison of fluorescence intensity. Asterisk indicate significant difference $(P<0.05)$. 
on both of cumulus cells and oocyte, and expression of protein was higher in mature oocytes than immature oocytes (Gall et al., 2004). Also, oocyte maturation was mediated by phosphorylation of MAPK through action of EGF and EGFR (Gall et al., 2005). Therefore, porcine oocyte maturation might be stimulated by a variety of signal pathway that are induced by action of EGF. And action of EGF might influence to change of organelle in oocyte such as Golgi apparatus and ER.

During process of oocyte maturation, the Golgi apparatus undergoes fragmentation and it is accumulated in the central part of the oocyte as dotted structure (Moreno et al., 2002). Moreover, Payne and Schatten (2003) had reported that the Golgi apparatus was translocalized into the ER during oocyte maturation of cow. Although the function of Golgi apparatus on oocyte maturation had been reported, regulation of this organelle by growth factors is not fully understood. In this results, fluorescence intensity of GM130, a marker of Golgi apparatus, was significantly higher in EGF-treated oocytes than EGF non-treated oocytes. These result show that expression of Golgi was stimulated by action of EGF, however, regulatory mechanism of Golgi apparatus by EGF stimulation during oocyte maturation is required more studies.

Sec61 complexes are transport protein that act as ER membrane protein translocator. And they are divided into three type subunit: alpha, beta, and gamma (Samuelson et al., 2000). Greenfield and High (1999) had reported that Sec61 complexes were located in both of the ER and ERGolgi intermediate compartment in COS1 cells, which are African green monkey kidney SV40 transformed cell line. And they suggested that Sec61 complexes were maintained in the ER membrane by a retrieval mechanism. These report indicated that Sec61 proteins play a potential role in transport between the ER and Golgi apparatus. In results of present study, expression of Sec61 $\beta$ protein was increased by EGF stimulation and localized in cytoplasm as well as GM-130 and EGFR expression. The relationship between
Sec61 $\beta$ and oocyte maturation was not reported. However, Sec61 complexes play a role in transport between Golgi apparatus and the ER that is closely related with oocyte maturation. Wang et al. (2010) reported that EGFR level in the nucleoplasm was reduced by knocking-down of Sec$61 \beta$. These reports suggest that Sec61 $\beta$ protein might influence to oocyte maturation via regulation of EGFR expression and mediation of transport between Golgi apparatus and the ER.

The relationship between expression of COPG2 and oocyte maturation was not reported as well as Sec61 $\beta$ protein. Cui et al. (2007) reported that expression of COPG2 mRNA was higher in 4-cell stage embryo and blastocyst than morula embryo. In in vitro produced bovine embryos, COPG2 mRNA was detected and it was expected that this gene function in embryonic developmental processes (Ripamonte et al., 2011). These effect of COPG2 on embryo development might be attributed by transport from Golgi apparatus to ER. Because transport between Golgi apparatus and ER is an important factor during oocyte maturation, COPG2 protein might be expected to play an important role in oocyte maturation processes. Despite of several studies associated with protein expression of ER/Golgi transport protein during oocyte maturation, transcriptional regulation of transport proteins were not reported.

In conclusion, we found that nuclear maturation of porcine oocytes were stimulated by EGF and stimulatory effect of EGF regulated expression of Golgi apparatus, Sec61 $\beta$, and COPG2. Especially, EGFR expression was down-regulated by absence of EGF stimulation and it might be related with expression of Sec61 $\beta$ protein that was regulated by EGF. This present study suggests that stimulatory effect of EGF is an important factor in porcine oocyte maturation and stimulation by EGF in oocytes could influenced not only intracellular signal transduction but also interaction between Golgi apparatus and ER that in mediated by transport protein. 


\section{ACKNOWLEDGEMENT}

This work was supported by the National Research Foundation of Korea (NRF) grant funded by the Korea Government (Ministry of Education) (2016R1D1A1B0393 1746).

\section{REFERENCES}

Bolamba D, Russ KD, Harper SA, Sandler JL, Durrant BS (2006) Effects of epidermal growth factor and hormones on granulosa expansion and nuclear maturation of dog oocytes in vitro. Theriogenology 65:1037-1047.

Conti M, Hsieh M, Park JY, Su YQ (2006) Role of the epidermal growth factor network in ovarian follicles. Mol Endocrinol 20:715-723.

Coskun S, Lin YC (1995) Mechanism of action of epidermal growth factor-induced porcine oocyte maturation. Mol Reprod Dev 42:311-317.

Cui XS, Li XY, Shen XH, Bae YJ, Kang JJ, Kim NH (2007) Transcription profile in mouse four-cell, morula, and blastocyst: Genes implicated in compaction and blastocoel formation. Mol Reprod Dev 74:133-143.

De La Fuente R, O’Brien MJ, Eppig JJ (1999) Epidermal growth factor enhances preimplantation developmental competence of maturing mouse oocytes. Hum Reprod 14:3060-3068.

De Prada JKN, Lee YS, Latham KE, Chaffin CL, Vande Voort CA (2009) Role for cumulus cell-produced EGFlike ligands during primate oocyte maturation in vitro. Am J Physiol Endocrinol Metab 296:E1049-E1058.

Eppig JJ (1996) Coordination of nuclear and cytoplasmic oocyte maturation in eutherian mammals. Reprod Fertil Dev 8:485-489.

Eppig JJ, Schultz RM, O'Brien M, Chesnel F (1994) Relationship between the developmental programs controlling nuclear and cytoplasmic maturation of mouse oocytes. Dev Biol 164:1-9.
Filardo EJ, Quinn JA, Franckelton Jr AR, Bland KI (2002) Estrogen action via the $\mathrm{G}$ protein-coupled receptor, GPR30: stimulation of adenylyl cyclase and cAMPmediated attenuation of the epidermal growth factor receptor-to-MAPK signaling axis. Mol Endocrinol 16:70-84.

Gall L, Boulesteix C, Ruffini S, Germain G (2005) EGFinduced EGF-receptor and MAP kinase phosphorylation in goat cumulus cells during in vitro maturation. Mol Reprod Dev 71:489-494.

Gall L, Chene N, Dahirel M, Ruffini S, Boulesteix C (2004) Expression of epidermal growth factor receptor in the goat cumulus-oocyte complex. Mol Reprod Dev 67: 439-445.

Greenfield JA, High S (1999) The Sec61 complex is located in both the ER and the ER-Golgi intermediate compartment. J Cell Sci 112:1477-1486.

Lindbloom S, Farmerie T, Clay C, Seidel G, Carnevale E (2008) Potential involvement of EGF-like growth factors and phosphodiesterases in initiation of equine oocyte maturation. Anim Reprod Sci 103:187-192.

Lonergan P, Carolan C, Van Langendonckt A, Donnay I, Khatir H, Mermillod P (1996) Role of epidermal growth factor in bovine oocyte maturation and preimplantation embryo development in vitro. Biol Reprod 54:1420 1429.

Mao L, Lou H, Lou Y, Wang N, Jin F (2014) Behaviour of cytoplasmic organelles and cytoskeleton during oocyte maturation. Reprod Biomed Online 28:284-299.

Mattioli M, Galeati G, Seren E (1988) Effect of follicle somatic cells during pig oocyte maturation on egg penetrability and male pronucleus formation. Gamete Res 20:177-183

Mendis C, Campbell K, Das R, Yang D, Jett M (2008) Effect of 5-lipoxygenase inhibitor MK591 on early molecular and signaling events induced by staphylococcal enterotoxin B in human peripheral blood mononuclear cells. FEBS J 275:3088-3098. 
Moreno RD, Schatten G, Ramalho-Santos J (2002) Golgi apparatus dynamics during mouse oocyte in vitro maturation: effect of the membrane trafficking inhibitor brefeldin A. Biol Reprod 66:1259-1266.

Payne C, Schatten G (2003) Golgi dynamics during meiosis are distinct from mitosis and are coupled to endoplasmic reticulum dynamics until fertilization. Dev Biol 264:50-63.

Procházka R, Sršeň V, Nagyová E, Miyano T, Flechon JE (2000) Developmental regulation of effect of epidermal growth factor on porcine oocyte-cumulus cell complexes: Nuclear maturation, expansion, and F-actin remodeling. Mol Reprod Dev 56:63-73.

Ripamonte P, Mesquita LG, Cortezzi SS, de Carvalho Balieiro JC, Fonseca Merighe GK, Watanabe YF, Caetano AR, Meirelles FV (2011) Differential gene expression and developmental competence in in vitro produced bovine embryos. Zygote 20:281-290.

Samuelson JC, Chen M, Jiang F, Möller I, Wiedmann M, Kuhn A, Phillips GJ, Dalbey RE (2000) YidC mediates membrane protein insertion in bacteria. Nature 406: 637-641.

Shimada M, Hernandez-Gonzalez I, Gonzalez-Robayna I, Richards JS (2006) Paracrine and autocrine regulation of epidermal growth factor-like factors in cumulus oo- cyte complexes and granulosa cells: key roles for prostaglandin synthase 2 and progesterone receptor. Mol Endocrinol 20:1352-1365.

Steeves T, Gardner D (1999) Metabolism of glucose, pyruvate, and glutamine during the maturation of oocytes derived from pre-pubertal and adult cows. Mol Reprod Dev 54:92-101.

Sutton-McDowall ML, Gilchrist RB, Thompson JG (2005) Effect of hexoses and gonadotrophin supplementation on bovine oocyte nuclear maturation during in vitro maturation in a synthetic follicle fluid medium. Reprod Fertil Dev 17:407-415.

Uhm SJ, Gupta MK, Yang JH, Chung HJ, Min TS, Lee HT (2010) Epidermal growth factor can be used in lieu of follicle-stimulating hormone for nuclear maturation of porcine oocytes in vitro. Theriogenology 73:1024-1036. Wang YN, Yamaguchi H, Huo L, Du Y, Lee HJ, Lee HH, Wang H, Hsu JM, Hung MC (2010) The translocon Sec61 $\beta$ localized in the inner nuclear membrane transports membrane-embedded EGF receptor to the nucleus. J Biol Chem 285:38720-38729.

Yarden Y (2001) The EGFR family and its ligands in human cancer: signalling mechanisms and therapeutic opportunities. Eur J Cancer 37:3-8. 